\title{
Masa corporal y calidad mecánica ósea. Influencia de modificadores ambientales
}

\section{Body mass and bone mechanical quality. Environmental modifiers influence}

\author{
Christian E. Lezón ${ }^{1}$, Clarisa Bozzini ${ }^{1}$, Patricia M. Pintos ${ }^{1}$, Alan Agüero-Romero ${ }^{1}$, Graciela M. Champin ${ }^{1}$, \\ SILVIA M. FRIEDMAN ${ }^{2}$ Y PATRICIA M. BOYER ${ }^{1 *}$
}

${ }^{1}$ Facultad de Odontología, Cátedra de Fisiología; ${ }^{2}$ Facultad de Odontología, Cátedra de Bioquímica General y Bucal. Universidad de Buenos Aires, Buenos Aires, Argentina

\section{RESUMEN}

Se evaluó la eficacia del propranolol (P) en la adecuación de la aptitud mecánica femoral en hipoxia en un modelo animal de retraso crónico del crecimiento de origen nutricional. Las ratas fueron distribuidas en dos grupos: normoxia $(\mathrm{Nx})$ e hipoxia $(\mathrm{Hx})$. Los grupos de $\mathrm{Nx}$ e Hx fueron subdivididos en: a) control ( $\mathrm{NxC}$ y $\mathrm{HxC}$ ), alimentados ad libitum; b) con restricción crónica de alimento del $20 \%(\mathrm{RC})$ (NxRC y HxRC); c) que recibieron alimentación ad libitum y $\mathrm{P}(\mathrm{NxCP}, \mathrm{HxCP})$, y d) con RC y $\mathrm{P}$ (NxRCP y $\mathrm{HxRCP}$ ), durante 28 días. En fémur se evaluaron las propiedades biomecánicas. La RC y la Hx produjeron un efecto negativo sobre el crecimiento corporal, la masa y las propiedades estructurales y geométricas óseas, siendo más evidentes en HxRC $(p<0.05)$. Las propiedades materiales resultaron similares entre grupos $(p>0.05)$. El $P$ revirtió los efectos negativos de la RC de alimento en la $\mathrm{Hx}$ hasta el nivel de HxCP. La reversibilidad del impacto negativo de la RC de alimento en $\mathrm{Hx}$ sobre el comportamiento biomecánico femoral en ratas en crecimiento podría ser atribuible, al menos en parte, a un efecto del fármaco sobre las células efectoras óseas con incremento de la rigidez estructural, relativa a la masa corporal.

Palabras clave: Biomecánica ósea. Crecimiento. Dieta. Restricción crónica. Hipoxia. Propranolol.

\begin{abstract}
This study evaluated the efficacy of propranolol $(P)$ in the adequacy of femoral mechanical competence in hypoxic conditions in an animal model of chronic food restriction. Rats were assigned into 2 groups: normoxia $(\mathrm{Nx})$ and hypoxia $(\mathrm{Hx})$. Nx and $\mathrm{Hx}$ groups were divided into: a) control ( $\mathrm{NxC}$ and $\mathrm{HxC}$ ) fed ad libitum; b) under chronic food restriction of $20 \%$ (CR) (NxCR and $\mathrm{HxCR}$; $\mathrm{C}$ ) fed ad libitum and $\mathrm{P}$ ip ( $\mathrm{NxCP}, \mathrm{HxCP})$, and d) under $\mathrm{CR}$ and $\mathrm{P}$ ip (NxCRP and HxCRP), for 28 days. Total body and femoral growth were recorded. In femur, fracture resistance, bone stiffness, cross-sectional and cortical areas, moment of inertia, modulus of elasticity and degree of mineralization were evaluated. CR and $\mathrm{Hx}$ induced negative effects on body growth, femoral and bone structural and geometric biomechanical properties; being more evident in $\mathrm{HxCR}(\mathrm{p}<0.05)$. The material properties were not affected $(p>0.05)$. In Hx, P could reverse the negative effects of chronic food restriction. The reversibility of the negative impact of chronic food restriction in $\mathrm{Hx}$ on femoral biomechanical behavior in growing rats could be attributable, at least in part, to an effect of the drug on bone cells with increased structural stiffness, relative to body mass.
\end{abstract}

Key words: Bone biomechanics. Growth. Diet. Chronic food restriction. Hypoxia. Propranolol.
Correspondencia:

*Patricia M. Boyer

E-mail: pboyer59@hotmail.com
Fecha de recepción: 18-10-2019

Fecha de aceptación: 06-11-2019

DOI: 10.24875/RME.19001958
Disponible en internet: $25-02-2020$ Rev Mex Endocrinol Metab Nutr. 2020;7:19-28

2462-4144/@ 2019 Sociedad Mexicana de Nutricion y Endocrinologia, AC. Publicado por Permanyer México. Este es un artículo open access bajo la licencia CC BY-NC-ND (http://creativecommons.org/licenses/by-nc-nd/4.0/). 


\section{INTRODUCCIÓN}

El patrón de crecimiento corporal global y el desarrollo del esqueleto, en particular, es el resultado del repertorio de instrucciones contenidas en el ácido desoxirribonucleico (ADN), de modificaciones epigenéticas y de la interacción del genomio con factores ambientales.

Los patrones normales de modificación epigenética pueden alterarse en la vida prenatal y/o posnatal como consecuencia de una falla en los mecanismos intrínsecos (defecto en la síntesis de las enzimas de remodelación de la cromatina, las ADN metiltransferasas y/o la histona desacetilasa) o por influencias ambientales tales como la exposición a agentes químicos, las radiaciones, la alimentación, la presión atmosférica y el consumo de fármacos, entre otros factores ${ }^{1}$.

En relación con el esqueleto, la capacidad resistiva de los huesos puede sufrir modificaciones en respuesta a estímulos «mecánicos» tales como el peso corporal y la actividad muscular, así como a estímulos «no mecánicos» de modulación sistémica y/o local del microambiente de las células óseas por acción de factores humorales, ambientales, farmacológicos, nutricionales y/o del sistema nervioso simpático ${ }^{2-5}$.

La modulación sistémica no direccional actúa activando o inhibiendo a las células efectoras óseas de todo el esqueleto, alterando la capacidad de respuesta de estas a los mediadores químicos liberados por el estímulo mecánico, y/o la capacidad mecanosensora de los osteocitos ${ }^{6}$.

El hueso como estructura de sostén es el resultado de la rigidez o resistencia a la deformación y, por ende, de la resistencia a la fractura. La rigidez ósea está determinada por la naturaleza fisicoquímica de su matriz calcificada y por la disposición arquitectónica de esa matriz en el espacio ${ }^{7-8}$.

La evidencia indica que la eficiencia estructural y mecánica esquelética está controlada por un mecanismo homeostásico de calidad ósea (mecanostato óseo) y determinada por las características esqueléticas del material duro y del diseño geométrico de este $^{9}$. En efecto, los huesos controlan su rigidez orientando la actividad de las células óseas en función del sensado direccional de la carga aplicada. Esta última es inferida por las deformaciones habituales determinadas por la gravedad y las contracciones musculares. Puede decirse, entonces, que la rigidez ósea adecuada es aquella necesaria y suficiente como para mantener la integridad física del hueso en respuesta a la carga, mientras que la resistencia a la fractura y la masa ósea alcanzadas constituyen el resultado de este mecanismo de control ${ }^{9}$.

A la estrecha relación entre la masa ósea y la masa corporal descrita se le suma otro factor mecánico, la actividad muscular, el cual afecta la competencia de los «huesos que soportan peso», como los axiales o apendiculares ${ }^{10}$. La concepción actual del esqueleto sostiene que está dinámicamente regulado por las deformaciones producidas por el uso muscular regional, con el propósito de optimizar la eficiencia mecánica del diseño arquitectónico en función de las cargas que soportan las regiones óseas. La contracción muscular constituye la carga fisiológica más importante ejercida sobre el hueso ${ }^{11}$.

En nuestro laboratorio hemos descrito un modelo experimental de «retraso crónico del crecimiento de origen nutricional» (RCCN) en ratas macho, el cual recrea un tipo de desnutrición observada en niños y adolescentes que consumen dietas inapropiadas deficientes en la energía global que impiden una ganancia de peso corporal y un crecimiento longitudinal adecuados ${ }^{12}$. Este modelo animal de RCCN se logró por administración crónica de una dieta equilibrada a ratas en crecimiento, en la que la restricción global fue del $20 \%$ comparada con los requerimientos energéticos de animales de igual edad ${ }^{12}$.

Estudios previos realizados en nuestro laboratorio demostraron que dichos animales presentaron un deterioro de las propiedades estructurales del fémur, con disminución de la aptitud mecánica en cuanto a la estructura para soportar fuerzas deformantes, resultado de modificaciones en la distribución arquitectónica del material ${ }^{13}$. Asimismo, se observó que los efectos combinados de una restricción de alimento en una atmósfera hipóxica indujeron una reducción adicional de la masa ósea y de su rigidez estructural en las ratas en crecimiento ${ }^{14}$. 
Dada la evidencia de un control $\beta$-adrenérgico de la masa ósea, de su distribución espacial y de la calidad mecánica intrínseca del material óseo ${ }^{15-17}$, el objetivo del presente trabajo fue evaluar la eficacia del $\beta$-bloqueante propranolol $(\mathrm{P})$ sobre la eficiencia del mecanostato óseo en optimizar la competencia mecánica ósea, en condiciones de hipoxia ambiental en el modelo animal de RCCN.

\section{MATERIAL Y MÉTODOS}

\section{Animales de experimentación}

Se emplearon ratas macho de la cepa Wistar desde el destete (25 días de edad; peso inicial: $62.23 \mathrm{~g} \pm 0.22$, [media \pm error estándar (ES)]) provenientes del Bioterio Central de la Facultad de Ciencias Exactas y Naturales de la Universidad de Buenos Aires. Los animales se ubicaron en jaulas galvanizadas con piso de malla con el propósito de mantener las condiciones de higiene y de evitar la coprofagia. La temperatura ambiental del bioterio fue de $21 \pm 1^{\circ} \mathrm{C}$ y la humedad del $50-60 \%$. Se respetaron los ciclos de luz-oscuridad para evitar alterar el ritmo de alimentación de los animales. Todos los experimentos fueron realizados cumpliendo las normas de los National Institutes of Health ${ }^{18}$ y aprobado por el Comité de Ética de la Facultad de Odontología de la Universidad de Buenos Aires (\#002/17).

\section{Dieta}

Los animales de experimentación se alimentaron con una dieta equilibrada para roedores presentada bajo la forma de cilindros prensados (Purina, Gilardoni S.A., Buenos Aires, Argentina), cuya composición centesimal ( $\mathrm{g} / 100 \mathrm{~g}$ de dieta) fue la siguiente: proteínas, 25.40; lípidos, 6.90; fibra, 4.80; Ca, 1.30; P, 0.80; cenizas, 5.50; agua, 8.30; dextrina csp, 100, y lisina disponible, 5.5/100 g de proteína.

\section{Diseño experimental}

Sesenta y cuatro ratas fueron distribuidas aleatoriamente en dos grupos: normoxia $(N x ; n=32)$ e hipoxia $(\mathrm{Hx}=$ exposición a $380 \mathrm{mmHg}, 23 \mathrm{~h} / \mathrm{d}$; $\mathrm{n}=32$ ). A su vez, los grupos $\mathrm{Nx}$ e $\mathrm{Hx}$ fueron divididos en cuatro subgrupos: a) control ( $\mathrm{NxC}$ y $\mathrm{HxC}$ ), que recibieron alimentación a libre demanda; $b$ ) con restricción crónica (RC) ( $\mathrm{NxRC}$ y HxRC), alimentados con un $80 \%$ de la dieta consumida por sus respectivos grupos control, $\mathrm{NxC}$ y $\mathrm{HxC}$, el día previo corregido por el peso corporal; $c$ ) que recibieron alimentación a libre demanda y $\mathrm{P}(\mathrm{NxCP}, \mathrm{HxCP})$, y d) con restricción crónica y $\mathrm{P}(\mathrm{N} \times \mathrm{RCP}$ y $\mathrm{HxRCP})$, durante 28 días.

El P (7 mg/kg/día) fue inyectado intraperitonealmente cinco días por semana durante cuatro semanas. La frecuencia de administración de $P$ fue elegida según estudios previos (Takeda, et al., 2002). Todas las ratas tuvieron libre acceso al agua de bebida. Se realizó el registro diario de la ingesta de alimento, y cada dos y cuatro días, el del peso y de la longitud corporales, respectivamente. La eutanasia de los animales se realizó por inyección intramuscular de $0.1 \mathrm{ml}$ de hidrocloruro de ketamina (100 mg/ml, Holliday Lab., Buenos Aires, Argentina)/100 g de peso corporal, y $0.02 \mathrm{ml}$ de xilazina $(100 \mathrm{mg} / \mathrm{ml}$, Konig Lab. Buenos Aires, Argentina) $/ 100 \mathrm{~g}$ de peso corporal, a las cuatro semanas experimentales (T4). De cada animal, se extrajeron los fémures, los cuales fueron pesados y medidos con un calibre digital. Ambos fémures se almacenaron a $-20{ }^{\circ} \mathrm{C}$ envueltos en gasa embebida en solución Ringer en bolsas plásticas selladas de acuerdo con Turner y Burr ${ }^{7}$ hasta la realización del ensayo biomecánico; en los fémures derechos se determinó el grado de mineralización porcentual.

\section{Determinación de la ingesta dietética durante el periodo experimental}

La ingesta de dieta se midió diariamente con una balanza Mettler PC 4000 con una precisión de \pm 1 $\mathrm{mg}$. La medida de consumo se determinó por diferencia de peso del comedero de dos días consecutivos. Este se expresó en g/100 g de rata/día.

\section{Evaluación antropométrica durante el período experimental}

Se midieron el peso ( $p$ ) y la longitud (L) corporales guardando un ayuno mínimo de dos horas y 
máximo de cuatro horas, previo a las mediciones. La frecuencia de medición de $p$ y $L$ fue diaria y semanal, respectivamente. El peso se midió utilizando una balanza Mettler PC 4000 con una precisión de $\pm 1 \mathrm{mg}$.

Para la medida de la longitud corporal, los animales se anestesiaron con una ligera anestesia (una mezcla de $2 \%$ de hidrocloruro de xilazina $[0.5 \mathrm{mg} / 100 \mathrm{~g}$ i.p. Konig Laboratories, Buenos Aires, Argentina] y $5 \%$ de hidrocloruro de ketamina $[5 \mathrm{mg} / 100 \mathrm{~g}$ i.p. Holliday-Scott S.A., Buenos Aires, Argentina]).

\section{Evaluación morfométrica del fémur}

La longitud del fémur se midió utilizando un calibre micrométrico digital con una precisión de $0.05 \mathrm{~mm}$. Se estimó la longitud femoral como la distancia entre el punto más proximal de la cabeza femoral y el punto más distal del cóndilo medial.

\section{Evaluación de las propiedades biomecánicas de la diáfisis femoral}

Se evaluó la calidad mecánica del hueso integrado como órgano mediante un ensayo mecánico de flexión a tres puntos en un equipo Instron modelo 4442 (Instron Corporation, Canton, MA, EE.UU.) ${ }^{7}$. Para realizar el estudio biomecánico los fémures fueron previamente descongelados por inmersión en solución fisiológica a temperatura ambiente. Cada fémur fue posicionado con la cara anterior orientada hacia abajo, sobre dos soportes separados por una distancia constante de $13 \mathrm{~mm}$ y equidistantes de los extremos epifisarios. Se aplicó una carga perpendicular al eje longitudinal del hueso a una velocidad constante de $5 \mathrm{~mm} / \mathrm{min}$ hasta la fractura del hueso ${ }^{8}$. Mediante el registro mecánico se obtuvieron las curvas carga (W)/deformación (d). A partir de dichas curvas se determinaron las siguientes variables mecánicas representativas del hueso femoral:

- Propiedades estructurales:

- Carga máxima de fractura (Wfmax, N), la que evalúa la resistencia efectiva del hueso a perder su integridad como órgano.
- Carga elástica límite (Wy, N), que define la capacidad máxima del hueso de deformarse al soportar cargas sin manifestar deformación plástica.

- Rigidez ósea (Wy/dy, máxima deformación elástica en el punto de cesión, $\mathrm{N} / \mathrm{mm}$ ), que evalúa la resistencia efectiva del hueso entero a ser deformado elásticamente por la acción de cargas.

- Propiedades geométricas. Mediante el uso de una sierra de diamante de baja velocidad (Isomet, Buehler, Lake Bluff, IL, EE.UU.) se realizaron cortes seccionales de $2 \mathrm{~mm}$ de espesor de la sección de fractura regularizada. Se registraron los diámetros horizontal y vertical, externo e interno de la sección de fractura mediante un calibre digital (Digimess, Ginebra, Suiza) y el apoyo de un estereomicroscopio (Stemi DV4 Stereomicroscope, Carl Zeiss Microimaging, Göttingen, Alemania). Se calcularon las siguientes propiedades geométricas:

- Área de sección transversa $\left(\mathrm{CSA}, \mathrm{mm}^{2}\right)$, estimada mediante la fórmula: $\pi / 4 . V . H$, donde $\mathrm{H}$ y $\mathrm{V}$ corresponden a los diámetros externos horizontal y vertical, respectivamente.

- Momento de inercia axial $\left(\mathrm{I}_{\mathrm{x}^{\prime}} \mathrm{mm}^{4}\right)$, propiedad geométrica que valoriza la eficiencia mecánica de la distribución espacial del material óseo en la sección transversal, con referencia al eje de flexión, que fue estimado mediante la fórmula: $\mathrm{I}_{\mathrm{x}}=\pi\left(\mathrm{V}^{3} \mathrm{H}-\mathrm{V}^{3} \mathrm{~h}\right) / 64$, donde $\mathrm{H}$ y V corresponden a los diámetros externos horizontal y vertical, respectivamente, y h y $v$ corresponden a los diámetros internos horizontal y vertical, respectivamente, de la sección transversal del hueso.

- Área medular $\left(\mathrm{MA}, \mathrm{mm}^{2}\right)$, estimada como $M A=\pi / 4 . v \cdot h$, donde $h$ y $v$ corresponden a los diámetros internos horizontal y vertical, respectivamente, de la sección transversal del hueso.

- Área cortical de la sección transversa $\left(\mathrm{A}, \mathrm{mm}^{2}\right)$, que se calculó por la diferencia entre CSA y MA.

1) Propiedades materiales:

- Módulo elástico de Young $\left(\mathrm{E}, \mathrm{N} / \mathrm{mm}^{2}\right)$, resistencia del material óseo a la deformación con independencia de su forma y dimensiones, que se calculó aplicando la ecuación $E=W y . I^{3 / 48}$ dy. $I_{x^{\prime}}$ donde $W y=$ máxima carga elástica, I =longitud 
Tabla 1. Consumo de alimento, tamaño corporal y morfometría ósea a las 4 semanas de tiempo experimental

\begin{tabular}{|c|c|c|c|c|c|c|c|c|c|}
\hline & $\mathrm{NxC}$ & $\mathrm{NxCP}$ & NxRC & NxRCP & $\mathrm{HxC}$ & $\mathrm{HxCP}$ & $\mathrm{HxRC}$ & HxRCP & $\mathrm{p}$ \\
\hline $\begin{array}{l}\text { Consumo de } \\
\text { alimento } \\
\text { g/100 g } \\
\text { peso/día }\end{array}$ & $11.85 \pm 0.36^{*}$ & $11.95 \pm 0.40^{*}$ & $9.46 \pm 0.28^{\dagger}$ & $9.56 \pm 0.31^{\dagger}$ & $9.95 \pm 0.35^{\dagger}$ & $9.56 \pm 0.29^{\dagger}$ & $7.96 \pm 0.31^{\ddagger}$ & $7.75 \pm 0.27^{\ddagger}$ & $<0.0001$ \\
\hline $\begin{array}{l}\text { Peso } \\
\text { corporal } \\
\text { a T0 (g) }\end{array}$ & $61.20 \pm 3.20^{*}$ & $62.80 \pm 4.50^{*}$ & $62.00 \pm 2.70^{*}$ & $61.80 \pm 3.20^{*}$ & $62.90 \pm 4.30^{*}$ & $62.10 \pm 3.80^{*}$ & $62.00 \pm 3.60^{*}$ & $63.00 \pm 3.40^{*}$ & 0.9999 \\
\hline $\begin{array}{l}\text { Peso } \\
\text { corporal } \\
\text { a T4 (g) }\end{array}$ & $302.40 \pm 5.60^{*}$ & $313.70 \pm 12.20^{*}$ & $173.70 \pm 3.30^{+}$ & $169.00 \pm 4.50^{\dagger}$ & $181.80 \pm 4.00^{\dagger}$ & $176.10 \pm 11.60^{\dagger}$ & $105.70 \pm 3.70^{\ddagger}$ & $101.80 \pm 4.90^{\ddagger}$ & $<0.0001$ \\
\hline $\begin{array}{l}\text { Longitud } \\
\text { corporal } \\
\text { a T0 }(\mathrm{cm})\end{array}$ & $15.1 \pm 0.1^{*}$ & $15.0 \pm 0.2^{*}$ & $14.9 \pm 0.9^{*}$ & $15.0 \pm 0.2^{*}$ & $15.3 \pm 0.4^{*}$ & $15.2 \pm 0.5^{*}$ & $15.0 \pm 0.6^{*}$ & $15.2 \pm 0.4^{*}$ & 0.9991 \\
\hline $\begin{array}{l}\text { Longitud } \\
\text { corporal } \\
\text { a T4 }(\mathrm{cm})\end{array}$ & $22.7 \pm 0.8^{*}$ & $22.4 \pm 0.7^{*}$ & $17.9 \pm 1.3^{\dagger}$ & $18.9 \pm 0.8^{\dagger}$ & $20.2 \pm 0.9^{\dagger}$ & $20.5 \pm 1.2^{\dagger}$ & $18.8 \pm 0.4^{\dagger}$ & $18.4 \pm 0.7^{\dagger}$ & 0.0002 \\
\hline $\begin{array}{l}\text { Peso del } \\
\text { fémur } \\
\text { a T4 (g) }\end{array}$ & $0.90 \pm 0.01^{*}$ & $0.91 \pm 0.01^{*}$ & $0.54 \pm 0.01^{\dagger}$ & $0.55 \pm 0.02^{\dagger}$ & $0.56 \pm 0.01^{\dagger}$ & $0.58 \pm 0.02^{+}$ & $0.49 \pm 0.01^{\ddagger}$ & $0.57 \pm 0.01^{\dagger}$ & $<0.0001$ \\
\hline $\begin{array}{l}\text { Longitud del } \\
\text { fémur } \\
\text { a T4 (mm) }\end{array}$ & $30.57 \pm 0.12^{*}$ & $31.03 \pm 0.31^{*}$ & $26.4 \pm 0.18^{\dagger}$ & $26.6 \pm 0.27^{\dagger}$ & $27.6 \pm 0.14^{\dagger}$ & $27.2 \pm 0.18^{\dagger}$ & $25.4 \pm 0.23^{\dagger}$ & $26.25 \pm 0.11^{\dagger}$ & $<0.0001$ \\
\hline
\end{tabular}

Los valores representan la media \pm error estándar ( $n=8$ animales/grupo).

Símbolos diferentes indican diferencias significativas entre grupos: ${ }^{*} ;{ }^{+} ;{ }_{;} ;$s

Nx: normoxia; Hx: hipoxia; C: control alimentado ad libitum; RC: restricción crónica de alimento del 20\%; P: propranolol; T4: cuatro semanas experimentales; T0: tiempo cero de experiencia.

del hueso, dy = máxima deformación elástica e $\mathrm{I}_{\mathrm{x}}=$ momento de inercia axial.

- Grado de mineralización porcentual. El fémur de cada animal se colocó en una estufa a $100{ }^{\circ} \mathrm{C}$ hasta peso constante y se obtuvo el peso seco. Luego este se colocó en una mufla a $600{ }^{\circ} \mathrm{C}$ durante $18 \mathrm{~h}$ y se obtuvo el peso de las cenizas. El grado de mineralización porcentual, que expresó el porcentaje de sustancia mineral en el hueso seco, se calculó como el peso de las cenizas/peso del hueso seco (\%).

\section{ANÁLISIS ESTADÍSTICO}

Los resultados se expresaron como media \pm ES. Los datos fueron analizados mediante análisis de varianza (ANOVA). A posteriori, se empleó el test de
Student-Neuman-Keuls de comparaciones múltiples. Las diferencias entre medias fueron consideradas significativas con un $p<0.05^{19}$. El análisis estadístico se realizó mediante el programa computarizado Graphpad Prism ${ }^{\circledR}$ version 5.03 (Graphpad Software, San Diego, CA, EE.UU.).

\section{RESULTADOS}

En la tabla 1 se presentan el consumo de alimento, el peso y la longitud corporales, el peso y la longitud del fémur de animales: control, alimentados a libre demanda $(C)$ y experimentales con alimentación restringida (RC) en normoxia e hipoxia con/sin P a T4.

El consumo de alimento fue significativamente menor en los animales hipóxicos respecto de sus controles normóxicos; el $\mathrm{P}$ no tuvo efecto sobre la 
ingesta de alimento, independientemente de la condición nutricional $(p<0.0001)$.

El crecimiento corporal global y femoral se afectó negativamente en las ratas $\mathrm{RC}$ comparado con sus respectivos controles $(p<0.001)$. El compromiso antropomorfométrico fue mayor en las ratas en condiciones de hipoxia (HxRC y HxRCP) $(p<0.001)$.

Al analizar el efecto de P sobre el peso corporal a T4 en los diferentes grupos experimentales respecto de sus controles ( $\mathrm{NxCP}$ vs. NxC, NxRCP vs. NxRC, $\mathrm{HxCP}$ vs. HxC y HxRCP vs. HxRC) no se encontraron diferencias significativas entre los grupos, independientemente de la condición nutricional y del entorno ambiental $(p=0.414, p=0.385, p=0.632$ y $p=0.525$, respectivamente). Resultados similares se obtuvieron en la longitud corporal ( $\mathrm{NxCP}$ vs. NxC, $\mathrm{NxRCP}$ vs. NxRC, HxCP vs. HxC y HxRCP vs. HxRC; $p=0.781, p=0.523, p=0.844$ y $p=0.627$, respectivamente).

En relación con el peso y longitud del fémur se obtuvieron diferencias significativas en el siguiente par de grupos: HxRCP vs. HxRC $(p=0.0001$ y $p=0.005$, respectivamente).

En las figuras 1-3 se muestran las propiedades estructurales, geométricas y materiales, respectivamente, del fémur de ratas control y experimentales a T4.

Los resultados biomecánicos del fémur muestran que las propiedades estructurales (carga máxima de fractura, carga en el límite elástico y rigidez ósea) de la media diáfisis se afectaron negativamente luego de cuatro semanas de restricción de alimento en las ratas $\mathrm{NxRC}$ respecto de los animales $\mathrm{NxC}$ $(p<0.0001)$ (Fig. 1)

Al analizar el área total y cortical de la sección transversa y el momento de inercia axial de la media diáfisis femoral, se observa que dichas variables geométricas fueron significativamente menores en el grupo NxRC respecto de las ratas $\mathrm{NxC}$ al final del periodo experimental ( $p<0.0001$ ) (Fig. 2).

Las propiedades biomecánicas del fémur resultaron significativamente menores en las ratas $\mathrm{HxC}$ y $\mathrm{HxRC}$ en relación con sus respectivos controles normóxi$\cos (\mathrm{NxC}$ y NxRC, respectivamente), siendo $\mathrm{HxRC}$ el grupo más afectado $(\mathrm{p}<0.0001)$ (Figs. 1 y 2 ).

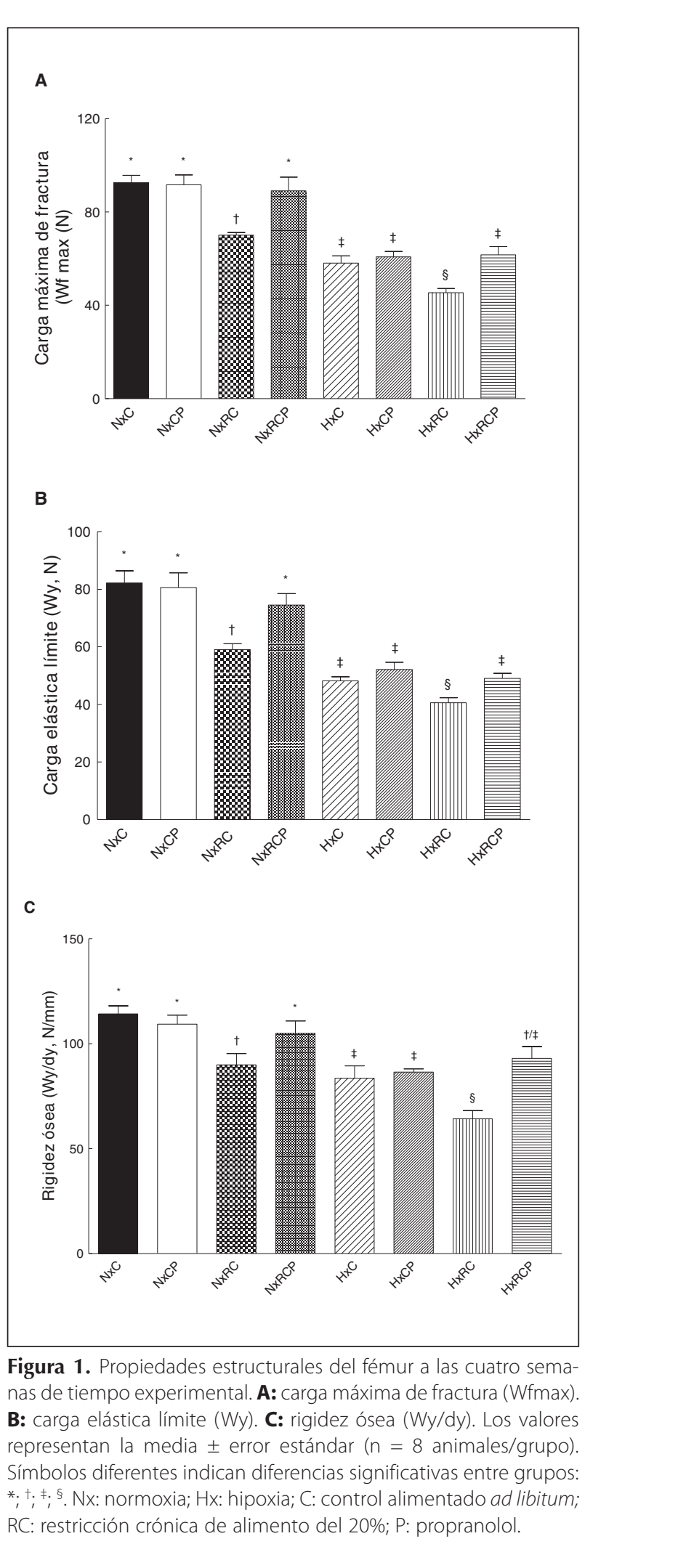




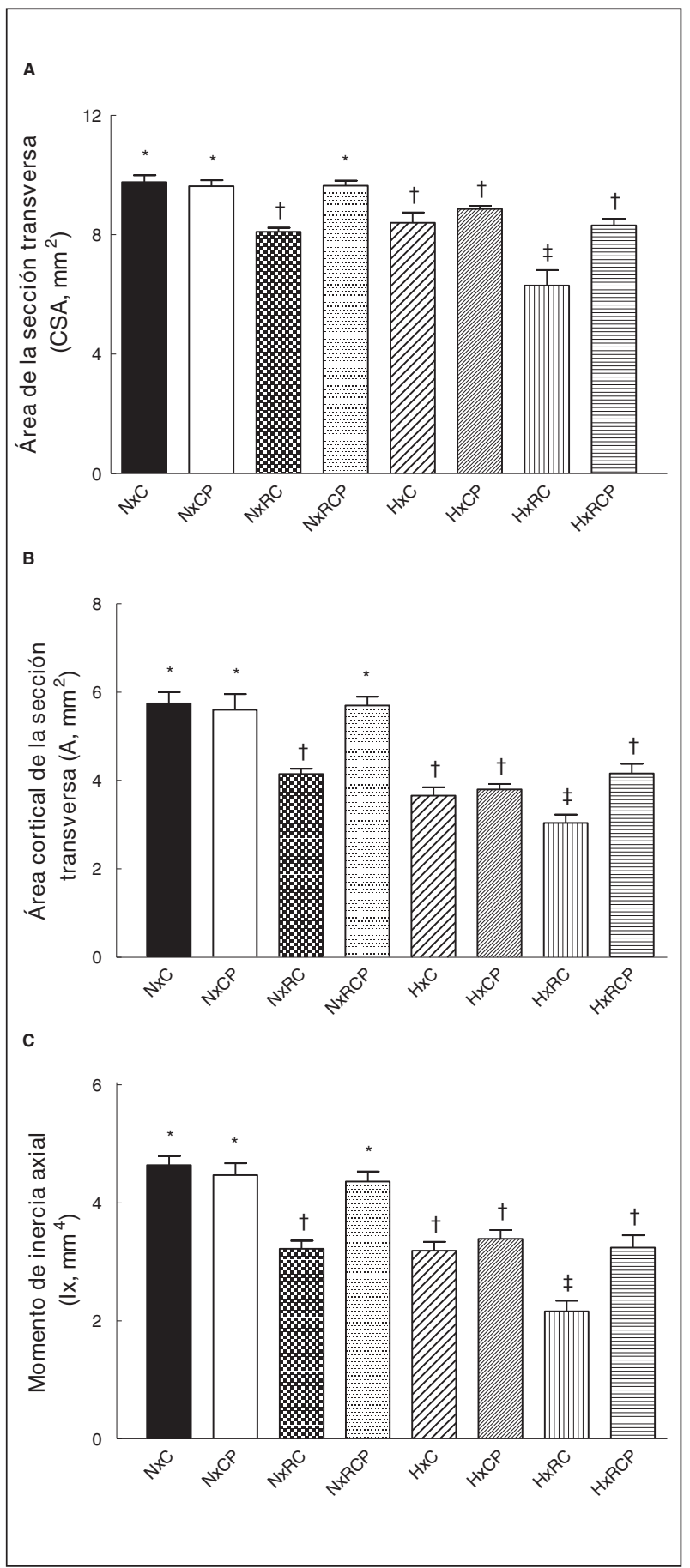

Figura 2. Propiedades geométricas del fémur a las cuatro semanas de tiempo experimental. A: área total de sección transversa (CSA). B: área cortical de sección transversa (A). C: momento de inercia (Ix). Los valores representan la media \pm error estándar ( $n=8$ animales/grupo). Símbolos diferentes indican diferencias significativas entre grupos: ${ }^{*} ;$ †; $\neq ;$; §. Nx: normoxia; Hx: hipoxia; C: control alimentado ad libitum; RC: restricción crónica de alimento del 20\%; P: propranolol.

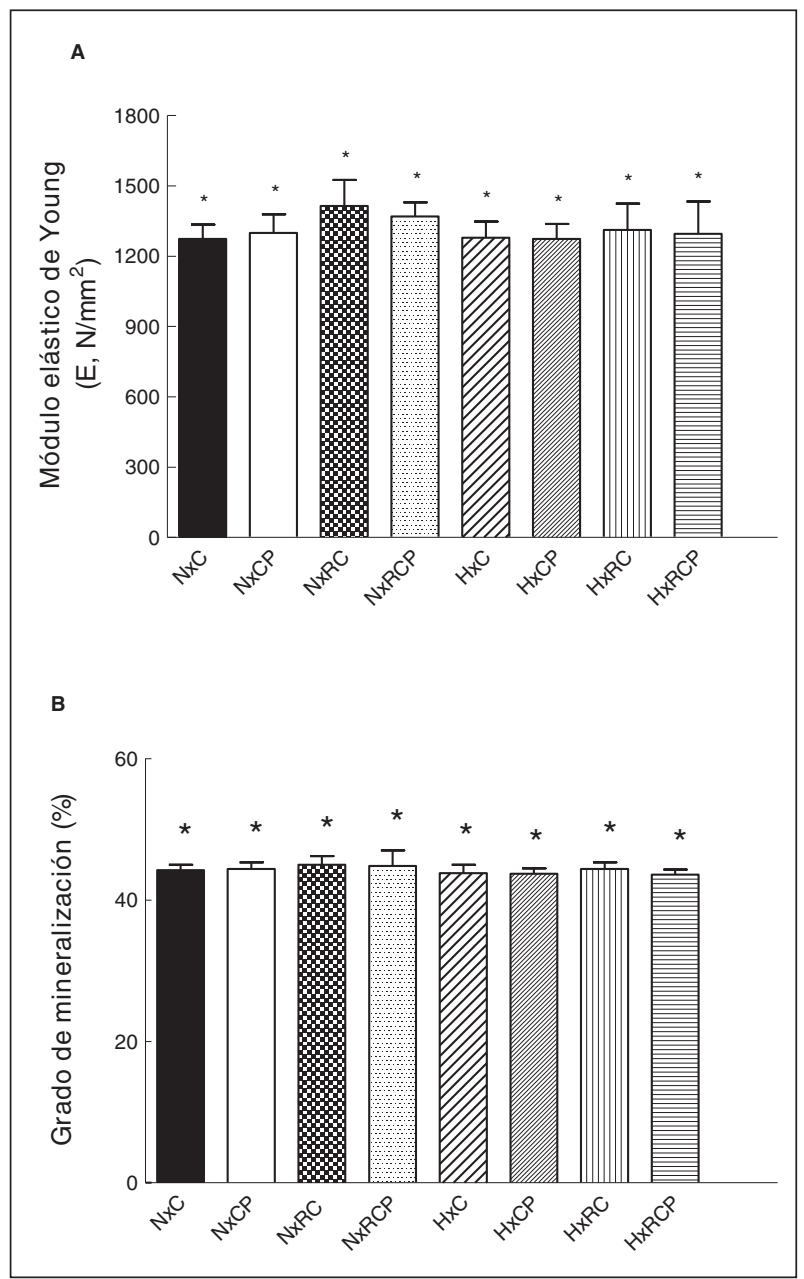

Figura 3. Propiedades materiales del fémur a las cuatro semanas de tiempo experimental. A: módulo elástico (E). B: grado de mineralización (\%). Los valores representan la media \pm error estándar ( $n=8$ animales/grupo). Símbolos diferentes indican diferencias significativas entre grupos:* * Nx: normoxia; Hx: hipoxia; C: control alimentado ad libitum; RC: restricción crónica de alimento del 20\%; P: propranolol.

Si bien el P no produjo cambios en las propiedades estructurales y geométricas del fémur de los animales $\mathrm{NxCP}$ y $\mathrm{HxCP}$, su administración indujo un incremento significativo de la competencia biomecánica femoral de los animales NxRCP y HxRCP, alcanzando los valores de sus respectivos controles ( $\mathrm{NxC}$ y HxC, respectivamente) $(p>0.05)$ (Figs.1 y 2$)$.

En relación con la calidad intrínseca del material óseo, inferida por el módulo de elasticidad y el grado de mineralización, no se observan diferencias significativas entre grupos a tiempo final ( $p>0.05)$ (Fig. 3). 


\section{DISCUSIÓN}

La aptitud mecánica del hueso está determinada por la rigidez que le confiere su estructura; definida por la naturaleza fisicoquímica de su matriz calcificada y por la disposición arquitectónica de esa matriz en el espacio ${ }^{7,8}$.

Con base en el control homeostásico de la rigidez estructural esquelética o eficiencia esquelética re-

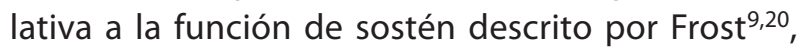
los huesos son estructuras dinámicamente reguladas en función de las deformaciones provocadas por el uso muscular regional. Como resultado de este tiene lugar un constante modelado o remodelado de la estructura ósea, a nivel cortical y trabecular, con el propósito de optimizar la eficiencia mecánica del diseño arquitectónico en función de la carga mecánica, representando la contracción muscular la carga fisiológica más importante ejercida sobre el hueso ${ }^{11}$.

El desarrollo esquelético del individuo responde a las instrucciones del programa genético del cigoto al que se le añaden modificaciones fisicoquímicas sobre el ADN, denominadas epigenéticas. Dichas modificaciones están destinadas a la activación o al silenciamiento de los genes, sin pérdida de la información genética del genoma individual del cigoto, y alteran la capacidad de expresión de algunos genes en las distintas etapas de desarrollo y según los diferentes tejidos del organismo en desarrollo ${ }^{21}$.

Puede decirse, entonces, que el fenotipo de un individuo refleja las modificaciones de las instrucciones potenciales de un genotipo en las distintas etapas del desarrollo del organismo, las que a su vez pueden afectarse por el entorno ambiental22, determinando, así, la alteración del fenotipo con la consecuente aparición de ciertas patologías ${ }^{23}$.

Existe evidencia de que la alimentación puede influir en las modificaciones epigenéticas del ADN y de las histonas. En efecto, es sabido que los grupos metilo son adquiridos de la dieta y donados al ADN por medio de la vía del folato y la metionina. El desequilibrio en la dieta puede conducir a una hipometilación, lo que puede provocar una inestabilidad genética y cromosómica ${ }^{1}$. Asimismo, la exposición a la altura, modelo fisiológico de hipoxia, induce una reducción del apetito y, por ende, una disminución del consumo de alimento, fenómeno conocido como "anorexia de la altura» ${ }^{24}$.

Si bien el mecanismo de modulación del apetito en la hipoxia aún no ha sido dilucidado completamente, existe evidencia de que la hipoxia crónica induce modificaciones endocrinas y metabólicas complejas. La anorexia de la altura podría ser la consecuencia de modificaciones en las concentraciones séricas de las hormonas reguladoras del apetito ${ }^{25}$.

La anorexia y, por ende, la reducción en la ingesta de alimento, produce un desequilibrio energético que conduce a la pérdida de peso y a cambios en la composición corporal ${ }^{26}$.

El presente estudio evidencia que la restricción de alimento indujo una marcada disminución de la velocidad de crecimiento en las ratas NxRC observada mediante los parámetros antropométricos. Este resultado podría deberse al efecto combinado de la restricción energética leve impuesta y de la cronicidad del estrés nutricional. Asimismo, los animales $\mathrm{HxC}$ presentaron un menor crecimiento corporal respecto de sus controles $\mathrm{Nx}$, resultado de una reducción de la velocidad de crecimiento de la masa corporal asociada a la hipofagia. Estos hallazgos son congruentes con la previa observación de un deterioro de la masa corporal asociado a la disminución del apetito en la altura ${ }^{26}$. Semejante comportamiento se observó en el crecimiento femoral.

La reducción de la tasa de crecimiento de las ratas HxC podría ser un mecanismo adaptativo de la masa corporal a la altura, con el fin de disminuir la demanda de oxígeno.

Por su parte, el $\mathrm{P}$ no pudo revertir los efectos negativos de la restricción de alimento y/o de la hipoxia ambiental sobre las variables antropométricas. Esto podría deberse a que el fármaco no tuvo efecto sobre el consumo de alimento o a que resultó ineficiente debido, al menos, a la dosis empleada y a la frecuencia de administración a lo largo del periodo experimental. Estos resultados concuerdan con los obtenidos por 
otros autores, quienes registraron cambios en la masa ósea sin modificaciones antropométricas frente a la administración del $\beta$-bloqueante ${ }^{27}$. Si bien no se observó un efecto global del $\mathrm{P}$ sobre la morfometría ósea, al evaluar su acción, en particular, sobre los animales en restricción de alimento en una atmósfera hipóxica, se evidencia el efecto beneficioso sobre la adquisición de masa ósea.

En relación con las propiedades estructurales de la media diáfisis, los resultados del presente estudio muestran que la capacidad resistiva femoral de ratas NxRC se afectó negativamente respecto de los animales $\mathrm{NxC}$, lo que resulta de una menor masa ósea con alteración del diseño arquitectónico. Más aún, la condición hipóxica produjo un mayor deterioro de la rigidez ósea incluso en condiciones de alimentación a libre demanda, siendo más acentuado el efecto negativo en condiciones de restricción de alimento.

En congruencia con las propiedades mecánicas analizadas, el área de la sección transversa y cortical, así como el momento de inercia de la sección transversa del fémur, fueron significativamente menores en los animales NxRC e HxRC en comparación a sus respectivos controles, lo que podría ser el resultado de una menor masa ósea y/o de la alteración de la distribución espacial de dicha masa.

En relación con los indicadores de las propiedades materiales, estos no fueron afectados por la restricción de alimento ni por la hipoxia. La ausencia de diferencias en las propiedades materiales en los animales subalimentados tratados con $\mathrm{P}$ (NxRCP y HxR$\mathrm{CP})$ sugiere que el incremento de la capacidad resistiva de la diáfisis femoral podría estar relacionado con un incremento de la masa ósea cortical y/o con una mayor eficiencia del diseño arquitectónico.

Dado que en estudios previos hemos observado que el grado de mineralización del colágeno relativo al peso femoral no se afectó por la restricción de alimento ${ }^{14}$, lo que es congruente con los resultados obtenidos en el presente estudio, y debido a que el peso del fémur de NxRCP y de HxRCP es semejante al de las ratas NxRC y HxRC, y significativamente menor respecto de $\mathrm{NxCP}$ y de $\mathrm{NxC}$, puede decirse que el mejoramiento del comportamiento biomecánico del fémur de ratas NxRCP y de HxRCP respecto de sus propios controles, podría ser el resultado de un efecto del tratamiento sobre el diseño macroarquitectónico del hueso más que sobre la masa ósea.

Con base en los conceptos propuestos por Frost relativos al mecanismo de autocontrol de la calidad mecánica del hueso ${ }^{9}$, el deterioro de la calidad ósea de las ratas NxRC y HxRC podría ser el resultado de una estimulación mecánica insuficiente por disminución del peso corporal y/o de la actividad de los músculos antigravitacionales, lo que determinará una pérdida neta de masa ósea como mecanismo de adaptación. Este comportamiento podría ser la consecuencia de una disminución del elevado costo metabólico del mantenimiento de una masa ósea, inadecuadamente excesiva para la actividad física del individuo ${ }^{28}$.

Asimismo, dada la evidencia de modificaciones en el fenotipo óseo en las ratas en crecimiento expuestas a factores ambientales tales como bajos niveles de presión parcial de oxígeno y/o a una ingesta inadecuada de alimento, los resultados del presente estudio sugieren que el deterioro de la calidad ósea que acompaña al retraso del crecimiento global, estaría asociado, al menos en parte, a una alteración de los patrones normales de modificación epigenética.

Dada la existencia de inervación noradrenérgica del hueso cortical ${ }^{5}$ y la de un control simpático del proceso de remodelado óseo ${ }^{29}$, en el presente estudio se observó que la administración de $\mathrm{P}$ en condiciones de restricción crónica de alimento optimizó la calidad ósea tanto en normoxia como en hipoxia, siendo la aptitud mecánica de las ratas NxRCP y HxRCP la de sus respectivos controles, condicionados por la presión parcial de oxígeno atmosférico.

Estos resultados sugieren que la acción del $\beta$-bloqueante sobre la eficiencia mecánica ósea está supeditada a la presión parcial de oxígeno ambiental; dicho factor ambiental prevalece sobre los efectos que resultan de modificaciones del patrón de alimentación en relación con la aptitud mecánica ósea. La reversibilidad del impacto negativo de la restricción de alimento en una atmósfera hipóxica sobre el comportamiento biomecánico femoral en ratas en crecimiento podría ser atribuible, al menos en parte, a un efecto del fármaco sobre las células efectoras óseas con incremento de la rigidez estructural relativa a la masa corporal alcanzada. 
FINANCIAMIENTO

El presente trabajo ha sido financiado por la Universidad de Buenos Aires UBACyT 2018-2020, 20020170100001BA.

\section{CONFLICTO DE INTERESES}

Los autores declaran no tener ningún conflicto de intereses.

\section{RESPONSABILIDADES ÉTICAS}

Protección de personas y animales. Todos los experimentos fueron realizados en animales de laboratorio, rata macho de la cepa Wistar, cumpliendo las normas del "National Institutes of Health, Laboratory animal welfare: Public Health Service policy on humane care and use of laboratory animals by awardee institutions. 1985, revised 1990", y aprobado por el Comité de Etica de la Facultad de Odontología de la Universidad de Buenos Aires (\#002/17).

Confidencialidad de los datos. Los autores declaran que en este artículo no aparecen datos de pacientes.

Derecho a la privacidad y consentimiento informado. Los autores declaran que en este artículo no aparecen datos de pacientes.

\section{BIBLIOGRAFÍA}

1. Feil R. Environmental and nutritional effects on the epigenetic regulation of genes. Mutat Res. 2006;600:46-57.

2. Ferretti JL, Capozza R, Cointry G, Bozzini C, Alippi RM, Bozzini CE. Additive effects of dietary protein and energy deficiencies on diaphysis and bone tissue of rat femurs as determined by bending tests. Acta Physiol Pharmacol Ther Latinoam. 1991;41:253-62.
3. Boyer PM, Compagnucci GE, Olivera MI, Bozzini C, Roig MC, Compagnucci $C V$, et al. Bone status in an animal model of chronic sub-optimal nutrition: a morphometric, densitometric and mechanical study. $\mathrm{Br}$ Nutr. 2005;93:663-9.

4. Russell RG Pharmacological diversity among drugs that inhibit bone resorption. Curr Opin Pharmacol. 2015;22:115-30.

5. Elefteriou F. Neuronal signaling and the regulation of bone remodeling Cell Mol Life Sci. 2005;62:2339-49.

6. Frost HM. The mechanostat: A proposed pathogenic mechanism of osteoporoses and the bone mass effects of mechanical and nonmechanical agents. Bone Miner. 1987;2:73-85.

7. Turner $\mathrm{CH}$, Burr DB. Basic biomechanical measurements of bone: a tutorial. Bone. 1993;14:595-608.

8. Ferretti JL. Biomechanical properties of bones. Osteoporosis and Bone Densitometry. Berlin: Springer Verlag; 1998.

9. Frost HM. Bone mass and the mechanostat: a proposal. Anat Rec 1987;219:1-9.

10. Frost HM. Strain and other mechanical influences of bone strength and maintenance. Curr Opin Orthop. 1987;8:60-70.

11. Ferretti JL, Cointry GR, Capozza RF, Capiglioni R, Chiappe MA. Analysis of biomechanical effects on bone and on the muscle-bone interactions in small animal models. J Musculoskelet Neuronal Interact. 2001;1:263-74.

12. Friedman SM, Rodriguez PN, Olivera MI, Bozzini C, Norese F, Gamba CA, et al. Nutrition dwarfism: longitudinal analysis of anthropometric and metabolic parameters in rats. Medicina (Buenos Aires). 1998;58:282-6.

13. Boyer PM, Compagnucci GE, Olivera MI, Bozzini C, Roig MC, Compagnucci $C V$, et al. Bone status in an animal model of chronic sub-optimal nutrition: a morphometric, densitometric and mechanical study. $\mathrm{Br}$ Nutr. 2005;93:663-9.

14. Lezon C, Bozzini C, Agûero Romero A, Pinto P, Champin G, Alippi RM, et al. Effect of chronic undernutrition on body mass and mechanical bone quality under normoxic and altitude hypoxic conditions. $\mathrm{Br} J$ Nutr. 2016;115:1687-95.

15. Lezón C, Olivera M, Bozzini C, Mandalunis P, Alippi R, Boyer P. Improved bone status by $\beta$-blocker propranolol in an animal model of nutritional growth retardation. Br J Nutr. 2009;101:1616-20.

16. Lezón CE, Pintos PM, Olivera MI, Bozzini C, Boyer PM. Effect of different doses of propranolol on bone structural and mechanical efficiency in an animal model of nutritional growth retardation. Endocrinol Nutr. 2012;59:9-20.

17. Pintos PM, Lezón CE, Bozzini C, Friedman SM, Boyer PM. Operationa mechanism modification of bone mechanostat in an animal model of nutritional stress: effect of propranolol. Rev Invest Clin. 2013;65: 39-51.

18. National Institutes of Health. Laboratory animal welfare: Public Health Service policy on humane care and use of laboratory animals by awardee institutions; 1985, revised 1990.

19. Sokal R, Rohlf J. Biometry: The principles and practice of statistics in biological research. $3^{\text {rd }}$ ed. New York: W.H. Freeman \& Company; 1995.

20. Frost HM. Perspectives: A proposed general model of mechanostat. Anat Rec. 1996;244:139-47.

21. Slotkin RK, Martienssen R. Transposable elements and the epigenetic regulation of the genome. Nat Rev Genet. 2007;8:272-85.

22. Jaenisch R, Bird A. Epigenetic regulation of gene expression: how the genome integrates intrinsic and environmental signals. Nature Genet 2003;33:S245-54.

23. Rodenhiser D, Mann M. Epigenetics and human disease: translating basic biology into clinical applications. CMAJ. 2006;174:341-8.

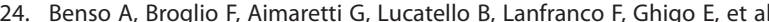
Endocrine and metabolic responses to extreme altitude and physical exercise in climbers. Eur J Endocrinol. 2007;157:733-40.

25. Quintero P, Milagro Fl, Campión J, Martínez JA. Impact of oxygen availability on body weight management. Med Hypotheses. 2010;74:901-7.

26. Westerterp KR, Kayser B. Body mass regulation at altitude. Eur J Gastroenterol Hepatol. 2006;18:1-3.

27. Takeda S, Elefteriou F, Levasseur R, Liu X, Zhao L, Parker KL, et al. Leptin regulates bone formation via the sympathetic nervous system. Cell. 2002;111:305-17.

28. Qin YX, Lam H, Ferreri S, Rubin C. Dynamic skeletal muscle stimulation and its potential in bone adaptation. J Musculoskelet Neuronal Interact. 2010;10:12-24.

29. Amling M, Takeda S, Karsenty G. A neuro (endo)crine regulation of bone remodeling. Bioessays 2000;22:970-5. 